\title{
Multi-Resolution Source Coding Theorems
}

\author{
Michelle Effros ${ }^{1}$ \\ Dept. of Electrical Eng. (136-93) \\ California Institute of Technology \\ Pasadena, CA 91125 USA \\ Email effros@caltech.edu
}

\begin{abstract}
For stationary sources on Polish alphabets, we describe the family of achievable rate and distortion vectors $\left(R_{1}, \ldots, R_{L}\right)$ and $\left(D_{1}, \ldots, D_{L}\right)$ for an $L$-resolution source code, where the description at the first resolution is given at rate $R_{1}$ and distortion $D_{1}$, the description at the second resolution includes both the first description and a refining description of rate $R_{2}$ and distortion $D_{2}$, and so on. We consider performance bounds for fixed- and variable-rate source codes on discrete-time stationary ergodic and stationary nonergodic sources for any integer number of resolutions $L \geq 1$. For $L>1$, the results extend previous results for discrete memoryless sources.
\end{abstract}

\section{INTRODUCTION}

In [1], Rimoldi describes the set of achievable ratedistortion vectors for $L$-resolution source coding on iid sources. We extend these results from iid sources to stationary sources on complete separable metric spaces (Polish alphabets), finding the optimal performance theoretically achievable by fixedand variable-rate multi-resolution codes. We use the variablerate and variable-distortion Lagrangian approach used for single-resolution codes in [2]. More details appear in [3].

\section{RESUlts}

Let $[A, \mu]=\left(A^{\infty}, \mathcal{A}^{\infty}, \mu, T\right)$ be a stationary dynamical system with Polish alphabet $A$. That is, $A$ is a complete separable metric space, $\mathcal{A}$ is the Borel $\sigma$-algebra generated by the open sets of $A, A^{\infty}$ is the set of one-sided sequences $x=\left(x_{1}, x_{2}, \ldots\right)$ from $A, \mathcal{A}^{\infty}$ is the $\sigma$-algebra of subsets of $A^{\infty}$ generated by finite-dimensional rectangles with components in $\mathcal{A}, T$ is the left shift operator on $A^{\infty}$, and $\mu$ is a measure on $\left(A^{\infty}, \mathcal{A}^{\infty}\right)$, stationary with respect to $T$.

Let $\rho\left(x_{1}, y_{1}\right)<\infty$ be a real-valued nonnegative distortion measure for $x_{1} \in A, y_{1} \in \hat{A}$, where $\hat{A}$ is an abstract reproduction alphabet. Assume that $\rho\left(x_{1}, y_{1}\right)$ is continuous in $x_{1}$ for each $y_{1} \in \hat{A}$ and that there exists a reference letter $y_{i}^{*} \in \hat{A}$ such that $E_{\mu} \rho\left(x_{1}, y_{1}^{*}\right)=d^{*}<\infty$. Define $\rho\left(x^{N}, y^{N}\right)=\sum_{i=1}^{N} \rho\left(x_{i}, y_{i}\right)$

Let $Q^{L, N}=\left(Q_{(\mathbf{1})}^{N}, \ldots, Q_{(L)}^{N}\right)$ be an $L$ resolution, fixed- or variable-rate, block quantizer with blocklength $N$. For any $\ell \in\{1, \ldots, L\}, Q_{(\ell)}^{N}$ maps $A^{N}$ onto some finite or countable set of codewords $\left\{y^{N}\right\}_{(\ell)}$ from $\hat{A}^{N}$. Together these codewords compose a codebook $\mathcal{C}_{(\ell)}^{N}=\left\{\left(y^{N},\left|y^{N}\right|\right)\right\}_{(\ell)}$ in which each codeword $y^{N}$ has an associated fixed- or variable-length binary description with length denoted $\left|y^{N}\right|$. We will assume that our description at the $(\ell-1)$ th resolution is embedded in the description at the $\ell$ th resolution. The codes are assumed

\footnotetext{
${ }^{1}$ Supported in part by NSF CAREER Award MIP-9501977, a grant from the Powell Foundation, and donations from Intel.
}

uniquely decodable, and if $Q^{L, N}$ is a fixed-rate code, then $\left|y^{N}\right|=c_{\ell}$ for all $y^{N} \in \mathcal{C}_{(\ell)}^{N}$.

Let $\mathcal{R}^{\mathrm{fr}, L}(\mu)$ and $\mathcal{R}^{\mathrm{vr}, L}(\mu)$ be the set of asymptotically achievable rate-distortion vectors $\left(R^{L}, D^{L}\right)$ for fixed- and variable-rate coding respectively. Then, by a time-sharing argument, $\mathcal{R}^{\mathrm{fr}, L}(\mu)$ and $\mathcal{R}^{\mathrm{vr}, L}(\mu)$ are convex sets, which can be totally characterized by their support functionals [4, p.135] $j^{\text {fr }}\left(\alpha^{L}, \beta^{L}, \mu\right)$ and $j^{\mathrm{vr}}\left(\alpha^{L}, \beta^{L}, \mu\right)$, here called the weighted fixed- and variable-rate operational rate-distortion functions, where $j^{\text {fr }}\left(\alpha^{L}, \beta^{L}, \mu\right)=\inf _{\left(r^{L}, d^{L}\right) \in \mathcal{R}^{\mathrm{fr}, L}(\mu)} \sum_{\ell=1}^{L}\left(\alpha_{\ell} d_{\ell}+\beta_{\ell} r_{\ell}\right)$ and $j^{\mathrm{vr}}\left(\alpha^{L}, \beta^{L}, \mu\right)=\inf _{\left(r^{L}, d^{L}\right) \in \mathcal{R}^{\mathrm{vr}, L}(\mu)} \sum_{\ell=1}^{L}\left(\alpha_{\ell} d_{\ell}+\beta_{\ell} r_{\ell}\right)$.

The Shannon weighted rate-distortion function is defined as $J\left(\alpha^{L}, \beta^{L}, \mu\right)=\inf _{N} J_{N}\left(\alpha^{L}, \beta^{L}, \mu\right)$, where the $N$ th-order weighted rate-distortion function is defined as $J_{N}\left(\alpha^{L}, \beta^{L}, \mu\right)=$ $\inf _{q} \frac{1}{N} \sum_{\ell=1}^{L}\left(\alpha_{\ell} E_{\mu^{N} q^{N}} \rho\left(X^{N}, \mathbf{Y}_{\ell}\right)+\beta_{\ell} I_{\mu q}\left(X^{N} ; \mathbf{Y}_{\ell} \mid \mathbf{Y}^{\ell-1}\right)\right)$ and $I\left(X^{N} ; Y_{\ell} \mid \mathbf{Y}^{\ell-1}\right)$ is a conditional mutual information associated with the test channel $q$ from $A^{N}$ to $\left(\hat{A}^{N}\right)^{L}$.

Stationary Ergodic Sources If $\mu$ is stationary and ergodic, then under the conditions on $A$ and $\rho$ given above, the following results hold.

Theorem $1 j^{\text {fr }}\left(\alpha^{L}, \beta^{L}, \mu\right)=J\left(\alpha^{L}, \beta^{L}, \mu\right)$.

Theorem $2 j^{\mathrm{vr}}\left(\alpha^{L}, \beta^{L}, \mu\right)=J\left(\alpha^{L}, \beta^{L}, \mu\right)$.

Theorem $3 \mathcal{R}^{\mathrm{fr}, L}(\mu)=\mathcal{R}^{\mathrm{vr}, L}(\mu)$.

Stationary Nonergodic Sources When $\mu$ is stationary and nonergodic, let $\left\{\mu_{x}: x \in A^{\infty}\right\}$ denote the ergodic decomposition of $\mu$. The ergodic decomposition exists by [5, Lemma 3.3.1, Lemma 2.4.1, Theorem 7.4.1]. Under the conditions given above, the following results hold.

Theorem $4 j^{\text {fr }}\left(\alpha^{L}, \beta^{L}, \mu\right)=\inf _{R^{L}} \int j^{\text {fr, } R^{L}}\left(\alpha^{L}, \beta^{L}, \mu_{x}\right) d \mu(x)$. Theorem $5 j^{\mathrm{vr}}\left(\alpha^{L}, \beta^{L}, \mu\right)=\int j^{\mathrm{vr}}\left(\alpha^{L}, \beta^{L}, \mu_{x}\right) d \mu(x)$.

Theorem $6 J\left(\alpha^{L}, \beta^{L}, \mu\right)=\int J\left(\alpha^{L}, \beta^{L}, \mu_{x}\right) d \mu(x)$.

Theorem $7 j^{\text {fr }}\left(\alpha^{L}, \beta^{L}, \mu\right) \geq J\left(\alpha^{L}, \beta^{L}, \mu\right)$.

Theorem $8 j^{\text {vr }}\left(\alpha^{L}, \beta^{L}, \mu\right)=J\left(\alpha^{L}, \beta^{L}, \mu\right)$.

Theorem $9 \mathcal{R}^{\mathrm{fr}, L}(\mu) \subseteq \mathcal{R}^{\mathrm{vr}, L}(\mu)$.

(We use $j^{\mathrm{fr}, R^{L}}\left(\alpha^{L}, \beta^{L}, \mu_{x}\right)$ to signify that each ergodic mode is forced to the same rate vector $R^{L}$.)

\section{REFERENCES}

[1] B. Rimoldi. Successive refinement of information: characterization of achievable rates. IEEE Transactions on Information Theory, 40(1):253-259, January 1994.

[2] M. Effros, P. A. Chou, and R. M. Gray. Variable-rate source coding theorems for stationary nonergodic sources. IEEE Transactions on Information Theory, IT-40(6):19201925, November 1994.

[3] M. Effros. Rate-distortion bounds for fixed- and variable-rate multi-resolution source codes. 1998. In review.

[4] D. G. Luenberger. Optimization by Vector Space Methods. John Wiley and Sons, New York, 1969.

[5] R. M. Gray. Probability, Random Processes, and Ergodic Properties. Springer-Verlag, New York, 1988. 